Implementation Strategy of a

Wellness Curriculum to Enhance

Attendance while Transitioning

the Format Due to COVID-19

Pandemic in a Pulmonary and

Critical Care Medicine Fellowship
RACHEL POTTER (D)

SAKSHI DUA (D)

*Author affiliations can be found in the back matter of this article
凹

Levy

Library

Press

\title{
ABSTRACT
}

Rationale: Burnout has been a growing area of concern impacting Pulmonary and Critical Care Medicine (PCCM) physicians both professionally and personally. Junior physicians, including medical fellows, have been identified as a population more at risk of developing burnout due to the stress and demands associated with training. Part of graduate medical education includes developing habits and skills a physician will use throughout their career. Fellowship training presents an ideal opportunity to provide wellness education and facilitate development of adaptive behaviors to cope with stress and decrease burnout. This need has been further emphasized as our trainees worked through a global pandemic which added a new set of stressors.

Methods: Pre-pandemic, a clinical social worker developed a wellness curriculum (WC) consisting of a variety of topics to address the components of burnout: emotional exhaustion, depersonalization, and reduced sense of personal achievement. Over a 4 year period, a WC of 1 hour facilitated discussions was established and trialed at different times, different days of the week, and frequencies (weekly vs biweekly vs monthly) within the PCCM fellowship program at a single institution. These meetings were held indoors in-person (IIP) pre-pandemic. As a result of COVID-19 pandemic, the WC was transitioned by switching the physical setting from IIP gatherings to either outdoor in person socially distant (OIP) gatherings (weather permitting) or virtual meetings online. Attendance was tracked for each wellness session.

Results: Over a 4 year period, pre-pandemic IIP attendance noticeably increased with modifications to time slots, day of the week, and frequency of wellness sessions. We found that: A) Wellness sessions held during lunch hour resulted in the higher attendance and engagement. B) The best day to hold these sessions was one that coincided with fellows' continuity clinic (Figure 1). C) The optimal frequency was monthly (rather than weekly or biweekly) (Figure 2). In contrast, after COVID-19 pandemic-

CORRESPONDING AUTHOR:

\section{Rachel Potter}

Icahn School of Medicine, US

Mount Sinai National Jewish Health Respiratory Institute, LCSW, US

rachel.potter@mssm.edu

\section{KEYWORDS:}

COVID-19; curriculum development; trainee wellness; pulmonary critical care medicine

\section{TO CITE THIS ARTICLE:} Potter R, Dua S. Identifying the Optimal Implementation Strategy of a Wellness Curriculum to Enhance Attendance while Transitioning the Format Due to COVID-19 Pandemic in a Pulmonary and Critical Care Medicine Fellowship. ISMMS Journal of Science and Medicine. 2021; 1(2): 13, pp. 1-3. DOI: https:// doi.org/10.29024/ijsm.60 
related adjustments, we found a significantly lower attendance to any sessions, whether OIP or virtual online. During the pandemic a slightly higher attendance at OIP sessions was noted compared with virtual online sessions (Figure 3).

Conclusion: Over a 4 year period, pre-pandemic, a WC was developed and adjusted based on the needs of PCCM fellows. We determined that the optimal time, day and frequency of a well-being curriculum was a lunch hour, after fellows' continuity clinic and monthly (rather than weekly or biweekly). However while transitioning the format due to COVID-19 pandemic we noted a sharp decline in overall attendance. Within the new adjustments, we did note a much better attendance at outdoor in-person socially distant meetings compared to virtual online format. Further work will be needed to determine the optimal strategy to enhance fellow attendance to wellness sessions in the "new normal."
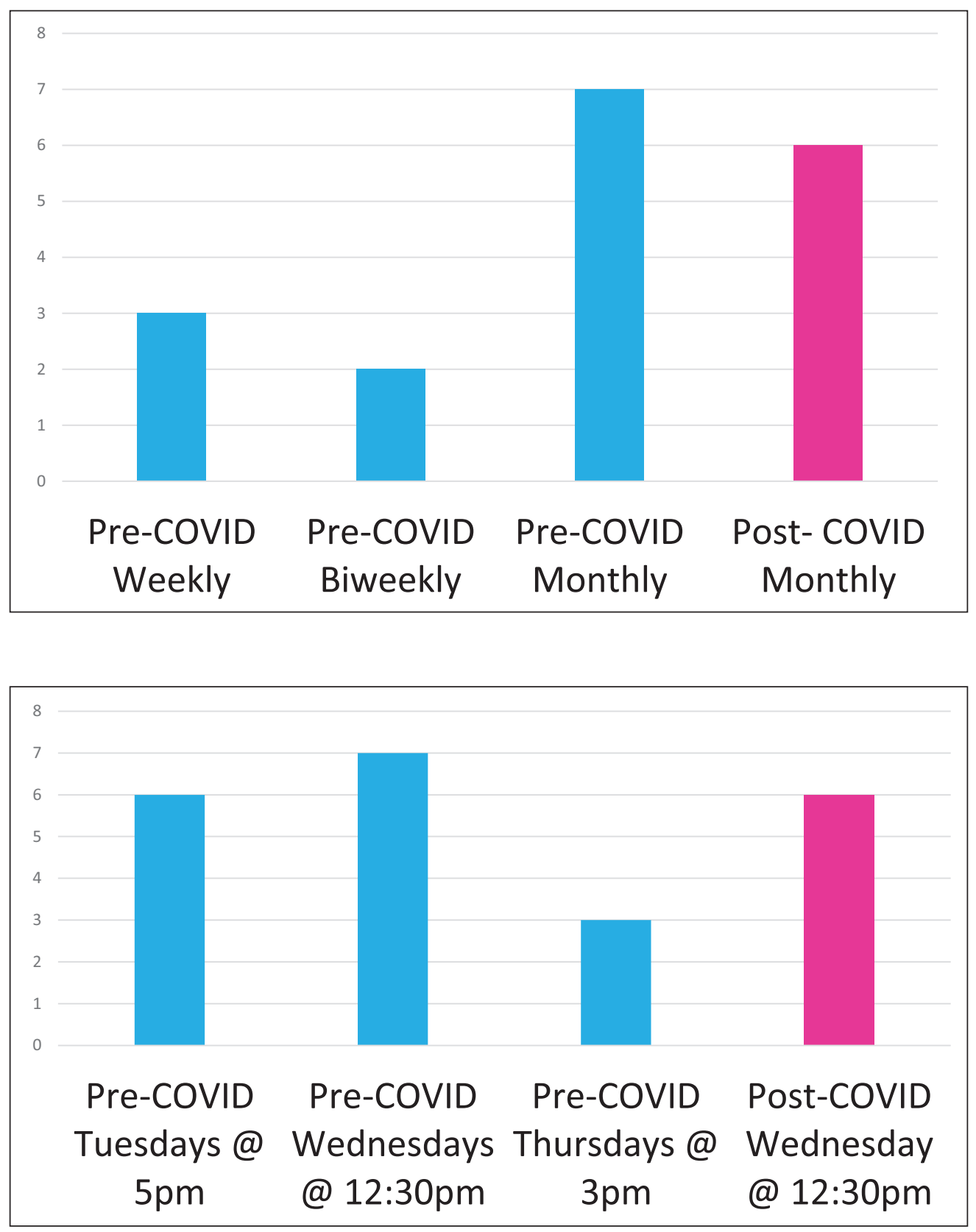

Figure 1 Average attendance by frequency of wellness session.

Figure 2 Average attendance by time and day of week. 


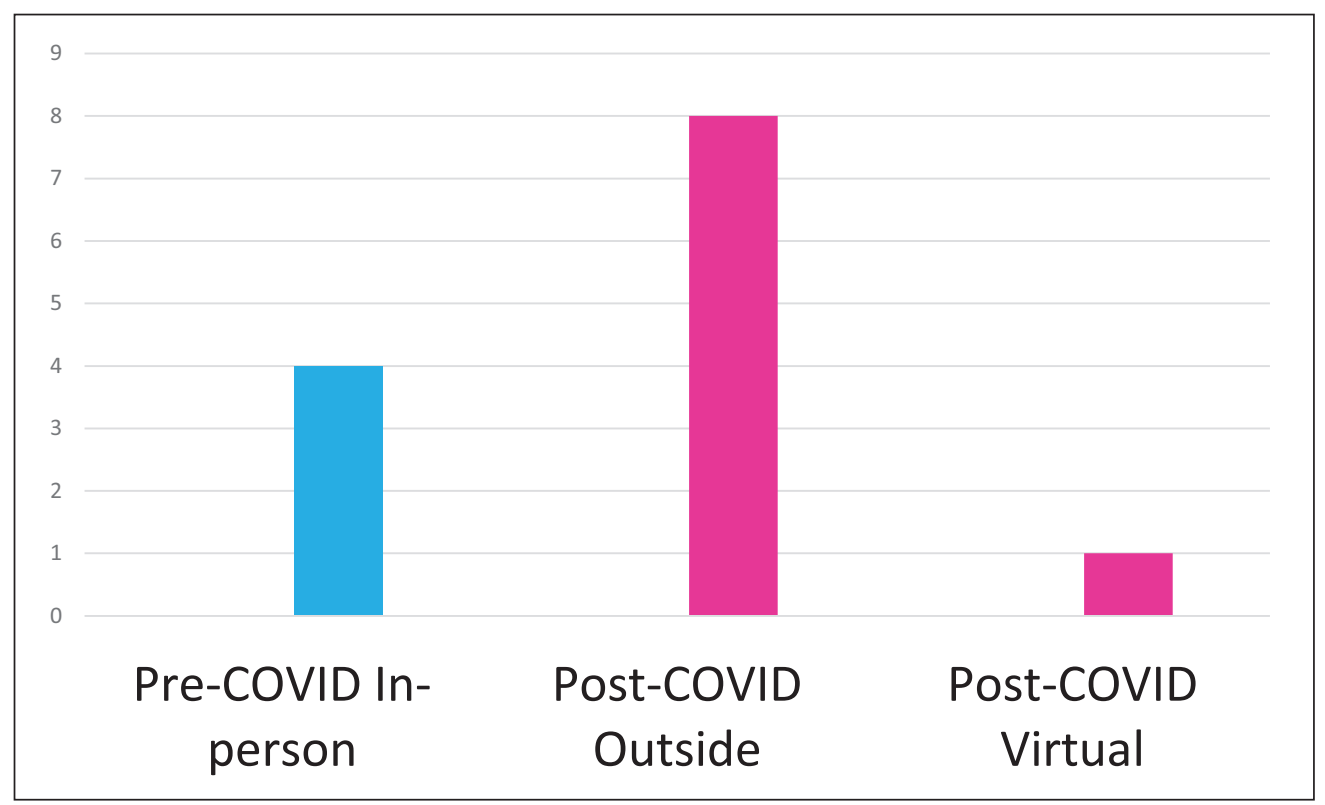

\section{COMPETING INTERESTS}

The authors have no competing interests to declare.

\section{AUTHOR AFFILIATIONS}

Rachel Potter (D) orcid.org/0000-0003-0828-2180

Icahn School of Medicine, US;

Mount Sinai National Jewish Health Respiratory Institute, LCSW, US

Sakshi Dua, MD (iD) orcid.org/0000-0002-8804-1055

Icahn School of Medicine, US
Figure 3 Average attendance by COVID group location.
TO CITE THIS ARTICLE: Potter R, Dua S. Identifying the Optimal Implementation Strategy of a Wellness Curriculum to Enhance Attendance while Transitioning the Format Due to COVID-19 Pandemic in a Pulmonary and Critical Care Medicine Fellowship. ISMMS Journal of Science and Medicine. 2021; 1(2): 13, pp. 1-3. DOI: https:// doi.org/10.29024/ijsm.60

Submitted: 16 April 2021

Accepted: 16 April 2021

Published: 10 June 2021

COPYRIGHT:

(c) 2021 The Author(s). This is an open-access article distributed under the terms of the Creative Commons Attribution 4.0 International License (CC-BY 4.0), which permits unrestricted use, distribution, and reproduction in any medium, provided the original author and source are credited. See http://creativecommons.org/ licenses/by/4.0\%.

ISMMS Journal of Science and Medicine is a peer-reviewed open access journal published by Levy Library Press. 